\title{
A retrospective cytohistological correlation of fine-needle aspiration cytology with classification by the Milan System for Reporting Salivary Gland Cytopathology
}

\author{
Ji Hyun Park ${ }^{1}$, Yoon Jin Cha ${ }^{1}$, Ja Yeong Seo ${ }^{1}$, Jae Yol Lim², Soon Won Hong ${ }^{1}$ \\ Departments of ${ }^{1}$ Pathology and ${ }^{2}$ Otorhinolaryngology, Gangnam Severance Hospital, Yonsei University College of Medicine, Seoul, Korea
}

\begin{abstract}
Background: Before publication of the new classification system named the Milan System for Reporting Salivary Gland Cytopathology (MSRSGC) in 2018, there was no standard classification for salivary gland lesions obtained by fine-needle aspiration (FNA). We therefore aimed to evaluate the diagnostic utility of this system by retrospectively reviewing FNA samples using the MSRSGC and to determine their risk of developing into neoplasms and becoming malignant. Methods: Retrospective slide review and classification of salivary gland FNAs obtained over a 6-year period (2013-2018) at a single center were performed by two pathologists. The risks of neoplasm and malignancy for each category also were calculated. Results: This study surveyed 374 FNAs (371 patients) performed over a sixyear period and selected 148 cases that included documented surgical follow-up (39.6\%). Among the surgically treated cases, the distributions of FNA categories were as follows: non-diagnostic (ND; $16.9 \%$ ), non-neoplastic (NN; $2.7 \%$ ), atypia of undetermined significance (AUS; 3.4\%), benign (BN; 54.7\%), salivary gland neoplasm of uncertain malignant potential (SUMP; 10.1\%), suspicious for malignancy (SM; 6.8\%), and malignant (M; 5.4\%). The risk of malignancy (ROM) was $24.0 \%$ for ND, $0 \%$ for NN, $40.0 \%$ for AUS, $2.5 \%$ for BN, $46.7 \%$ for SUMP, $100 \%$ for SM, and $87.5 \%$ for M. The overall diagnostic accuracy was $95.9 \%$ (142/148 cases). Conclusions: The newly proposed MSRSGC appears to be a reliable system for classification of salivary gland lesions according to the associated ROM.
\end{abstract}

Key Words: Salivary gland; Fine-needle aspiration; The Milan System for Reporting Salivary Gland Cytopathology; Risk of malignancy

Received: February 17, 2020 Revised: June 5, 2020 Accepted: June 9, 2020

Corresponding Author: Soon Won Hong, MD, PhD, Department of Pathology, Gangnam Severance Hospital, Yonsei University College of Medicine, 211 Eonju-ro, Gangnam-gu, Seoul 06273, Korea

Tel: +82-2-2019-3540, Fax: +82-2-2019-3540, E-mail: soonwonh@yuhs.ac

Salivary gland fine-needle aspiration (FNA) is a well-established, minimally invasive, and cost-effective procedure that rarely results in complications [1,2]. FNA provides crucial information for clinical management of tumors, such as by distinguishing between neoplastic and non-neoplastic lesions and benign and malignant lesions, as well as by providing samples for ancillary tests [3-7]. Clinical management and surgical interventions heavily depend on the information provided by FNA, along with clinical data and information obtained from imaging studies. It is crucial to differentiate between benign and malignant lesions. However, intratumoral heterogeneity and overlapping cytologic features of different salivary gland lesions hinder accurate subtyping of neoplasms [8-11]. Until recently, there has been no uniform reporting system for salivary gland lesions. A descriptive cytologic diagnosis without categorization can be confusing for clinicians, who require more accurate information to establish an effective management plan [9].

In an attempt to address these challenges, an international group of pathologists and clinicians developed a tier-based classification system with the support of the American Society of Cytopathology and the International Academy of Cytology that was designated the Milan System for Reporting Salivary Gland Cytopathology (MSRSGC) [12,13]. The goal of this classification system was to standardize a reporting system for salivary gland lesions and to provide guidelines for their clinical management. Like other similar reporting systems, such as the Bethesda System for reporting thyroid cytopathology, this new classification system offers a clinically valuable framework for conceptualizing salivary gland lesions [14]. The MSRSGC is composed of seven categories: non-diagnostic (ND), non-neo- 
plastic (NN), atypia of undetermined significance (AUS), benign neoplasm (BN), salivary gland neoplasm of uncertain malignant potential (SUMP), suspicious for malignancy (SM), and malignant $(\mathrm{M})$.

Thus far, few studies have demonstrated promising use of this system. Therefore, in this study, we retrospectively applied the MSRSGC to categorize salivary gland FNA samples from 2013-2018. The objective of this study was to evaluate the diagnostic accuracy of the MSRSGC framework and evaluate this system's utility as a tool for risk assessment of salivary gland lesions.

\section{MATERIALS AND METHODS}

\section{Research objective}

A retrospective search of the cytopathology database from the past six years (January 2013-December 2018) for salivary gland (all major and minor salivary glands) FNA specimens at Gangnam Severance Hospital, School of Medicine, Yonsei University was performed. Clinical data regarding age, sex, and location of the lesion, as well as the type of tumor, were collected from patient medical records. Follow-up histopathological reports were also obtained if available. From January 2013 to December 2018, 374 FNAs were performed, and 150 of these also underwent surgical resection. Among them, 148 cases were finally enrolled in this study; two cases were excluded as they did not meet the inclusion criteria.

The FNAs were performed via a direct percutaneous or transoral route using a 23-gauge needle. The smears were then fixed in 95\% ethanol for Papanicolaou staining, which was performed in the cytopathology laboratory.

\section{Slide review and categorization}

Blinded review of all FNA slides was carried out by two pathologists (J.H.P. and Y.J.C.), and each case was assigned to an MSRSGC category. When there was a diagnostic discrepancy, the two pathologists had a discussion to decide upon the best MSRSGC category. Category I cases were further divided into an inadequate group and a cyst-contents-only group. Matched slides of surgical specimens were also examined, and the histological diagnoses of these surgical specimens were categorized as $\mathrm{NN}, \mathrm{BN}$, or $\mathrm{M}$.

\section{Evaluation of risk of malignancy and risk of neoplasm}

Cytologic-histologic correlations were performed to determine risk of malignancy (ROM) and risk of neoplasm (RON).
The ROM was defined as the ratio between the number of FNAs and the number of surgically confirmed malignancies. Similarly, the RON was defined as the ratio between the number of FNAs and the number of neoplasms, including both benign and malignant neoplasms. The ROM and RON values were calculated for each MSRSGC category.

\section{RESULTS}

\section{Basal patient characteristics}

This study included a total of 148 cases. The clinicopathologic characteristics of the patients are shown in Table 1. Our study population was made up of 64 (43.2\%) male and 84 (56.8\%) female patients, with a median age of 49 years (range, 11 to 85 years). FNA was performed predominantly from the parotid gland $(n=120,81.1 \%)$. Thirty-four cases $(23.0 \%)$ were confirmed as $\mathrm{M}$ neoplasms by histological categorization. Their categorization according to the MSRSGC is shown in Fig. 1.

\section{Correlation between pathologic diagnosis and diagnosis based on MSRSGC categorization of FNA results}

The preoperative cytological diagnoses and histological follow-up results are listed in Table 2. There were 25 cases with category I FNAs: eight NN lesions, $11 \mathrm{BNs}$, and six M neoplasms. There were four cases of category II FNAs: two NN lesions and two BNs. Among the five cases with category III FNAs, three involved pleomorphic adenomas and the other two were one

Table 1. Basal characteristics of patients

\begin{tabular}{lc}
\hline Characteristic & No. $(\%)$ \\
\hline Sex & \\
Male & $64(43.2)$ \\
Female & $84(56.8)$ \\
Age (yr), mean \pm SD (range) & $50.0 \pm 15.1(11-85)$ \\
Location & \\
Parotid & $120(81.1)$ \\
Submandibular & $28(18.9)$ \\
MSRSGC category & \\
I & $26(16.9)$ \\
II & $4(2.7)$ \\
III & $5(3.4)$ \\
IV-A & $81(54.7)$ \\
IV-B & $15(10.1)$ \\
V & $10(6.8)$ \\
VI & $8(5.4)$ \\
Pathologic diagnosis category & \\
Non-tumor lesion & $11(7.4)$ \\
Benign neoplasm & $103(69.6)$ \\
Malignant neoplasm & $34(23.0)$ \\
\hline
\end{tabular}

MSRSGC, Milan System for Reporting Salivary Gland Cytopathology. 

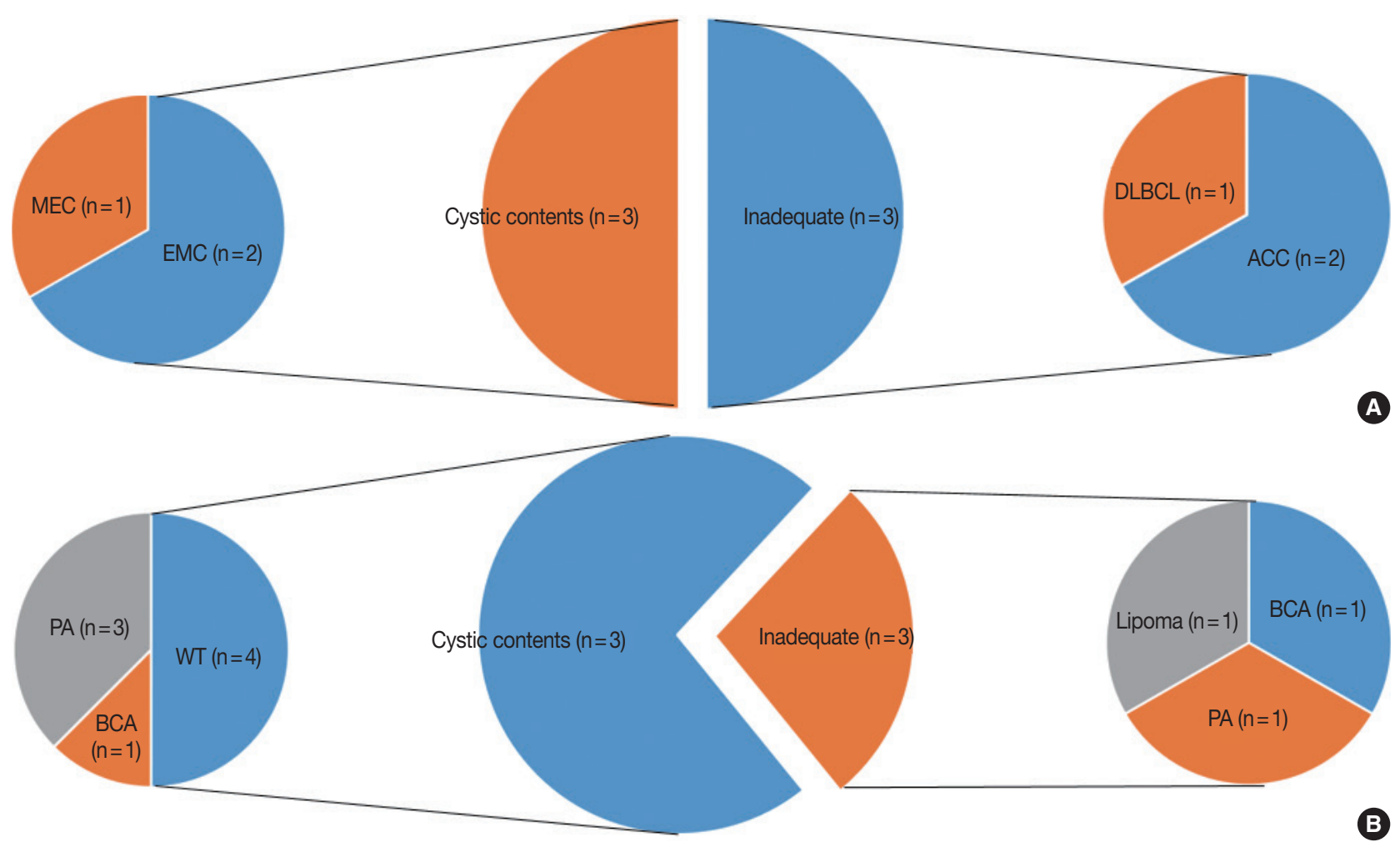

Fig. 1. Distribution of malignant (A) and benign (B) neoplasms in category I fine-needle aspiration samples. PA, pleomorphic adenoma; WT, Warthin tumor; MEC, mucoepidermoid carcinoma; EMC, epithelial myoepithelial carcinoma; ACC, acinic cell carcinoma; DLBCL, diffuse large B-cell lymphoma; BCA, basal cell adenoma.

case each of metastatic breast cancer in the intraglandular lymph node and diffuse large B-cell lymphoma. Notably, our results showed that $97.5 \%(79 / 81)$ of category IV-A cases were BN. However, the FNAs of one mucoepidermoid carcinoma and one epithelial myoepithelial carcinoma were placed into category IV-A. Among the category IV-B cases, seven were BN and seven were $\mathrm{M}$ neoplasms. In contrast, all category $\mathrm{V}$ cases involved malignant tumors, half of which were epithelial myoepithelial carcinomas. Among the eight category VI FNA cases, seven involved malignant neoplasms, and one was an atypical pleomorphic adenoma.

\section{Further analysis of category I cases}

Of all FNA cases that were followed by surgical resection, category I cases accounted for 17\% (25/148). Of the 25 category I cases, 22 were located in the parotid gland, which is significantly higher than the remaining three cases that involved the submandibular gland. The lesions diagnosed as category I in the submandibular gland included fibrocalcific nodules, IgG4-related disease, and chronic sialadenitis. There were six category I FNA cases with lesions diagnosed as malignant tumors, which included two acinic cell carcinomas, two epithelial myoepithelial carci- nomas, one diffuse large B-cell lymphoma, and one mucoepidermoid carcinoma. (Fig. 1). Moreover, there were 11 category I FNA cases with lesions diagnosed as benign tumors, including four pleomorphic adenomas, four Warthin tumors, two basal cell adenomas, and one lipoma. Only cystic contents without cells were aspirated in four cases involving Warthin tumors and three cases involving pleomorphic adenomas (Fig. 1B).

\section{Risk stratification and comparisons with previous studies}

We found that the RON was $68.0 \%$ for ND, $50.0 \%$ for NN, $100 \%$ for AUS, $100 \%$ for BN, $93.3 \%$ for SUMP, $100 \%$ for $\mathrm{SM}$, and $100 \%$ for $\mathrm{M}$. The corresponding ROM values were $24.0 \%$ for ND, $0 \%$ for NN, $40.0 \%$ for AUS, $2.5 \%$ for BN, $46.7 \%$ for SUMP, $100 \%$ for SM, and $87.5 \%$ for M. A summary of the ROM values obtained in the current study and those proposed by the MSRSGC and other studies is shown in Table 3.

\section{Discrepant cases}

A discrepancy between FNA and pathological diagnoses was observed in six cases (Table 4). In the NN FNA group (two cases), one case was diagnosed as a Warthin tumor and the other as a sialolipoma. Among the two cases classified as BNs using the 
FNA samples, both were reported as malignant on resection (mucoepidermoid carcinoma and epithelial myoepithelial carcinoma). Moreover, among the cases classified as SUMPs, one was later diagnosed as a reactive lymph node (paracortical hyperplasia). Finally, among the cases categorized as malignant using
FNA samples, one was diagnosed as atypical pleomorphic adenoma upon histological follow-up (Fig. 2). The diagnostic accuracy achieved using FNA samples was $95.8 \%$ (115/120 cases) for the parotid gland and $96.4 \%$ (27/28 cases) for the submandibular gland. Thus, the overall diagnostic accuracy using FNA

Table 2. Categorization of pathological diagnoses of fine-needle aspirations based on the Milan System for Reporting Salivary Gland Cytology (MSRSGC)

\begin{tabular}{|c|c|c|c|}
\hline \multirow{2}{*}{ MSRSGC category } & \multicolumn{3}{|c|}{ Pathological diagnosis } \\
\hline & Non-neoplastic $(n=11)$ & Benign neoplasm ( $n=103)$ & Malignant neoplasm $(n=34)$ \\
\hline $\mathrm{I}(\mathrm{n}=25)$ & $\begin{array}{l}\text { Lymphoepithelial cyst }(n=4) \\
\text { IgG4-related disease }(n=2) \\
\text { Chronic sialadenitis }(n=1) \\
\text { Fibrocalcific nodule }(n=1)\end{array}$ & $\begin{array}{l}\text { WT }(n=4) \\
\text { PA }(n=4) \\
\text { Lipoma }(n=1) \\
\text { BCA }(n=2)\end{array}$ & $\begin{array}{l}\operatorname{ACC}(n=2) \\
\operatorname{EMC}(n=2) \\
\operatorname{DLBCL}(n=1) \\
\operatorname{MEC}(n=1)\end{array}$ \\
\hline$\|(n=4)$ & $\begin{array}{l}\text { Reactive lymph node }(n=1) \\
\text { Epidermal cyst }(n=1)\end{array}$ & $\begin{array}{l}\text { WT }(n=1) \\
\text { Sialolipoma }(n=1)\end{array}$ & None \\
\hline III $(n=5)$ & None & $\mathrm{PA}(\mathrm{n}=3)$ & $\begin{array}{l}\text { Metastatic carcinoma }(n=1) \\
\text { DLBCL }(n=1)\end{array}$ \\
\hline IV-A $(n=81)$ & None & $\begin{array}{l}\text { PA }(n=48) \\
\text { WT }(n=27) \\
\text { Oncocytoma }(n=1) \\
\text { BCA }(n=1) \\
\text { Myoepithelioma }(n=1) \\
\text { Atypical PA }(n=1)\end{array}$ & $\begin{array}{l}\operatorname{MEC}(n=1) \\
\operatorname{EMC}(n=1)\end{array}$ \\
\hline IV-B $(n=15)$ & Reactive lymph node $(n=1)$ & $\begin{array}{l}\text { PA }(n=4) \\
\text { Oncocytoma }(n=1) \\
\text { Myoepithelioma }(n=1) \\
\text { Hemangioma }(n=1)\end{array}$ & $\begin{array}{l}\text { MEC }(n=3) \\
\text { EMC }(n=2) \\
\text { ACC }(n=1) \\
\text { Carcinoma ex PA }(n=1)\end{array}$ \\
\hline$V(n=10)$ & None & None & $\begin{array}{l}\operatorname{MEC}(n=5) \\
\operatorname{AdCC}(n=2) \\
\operatorname{ACC}(n=1) \\
\operatorname{DLBCL}(n=1) \\
\operatorname{SqcC}(n=1)\end{array}$ \\
\hline $\mathrm{VI}(\mathrm{n}=8)$ & None & Atypical PA $(n=1)$ & $\begin{array}{l}\text { MEC }(n=3) \\
\text { ACC }(n=2) \\
\text { Adenocarcinoma, NOS }(n=1) \\
\text { Metastatic melanoma }(n=1)\end{array}$ \\
\hline
\end{tabular}

WT, Warthin tumor; ACC, acinic cell carcinoma; PA, pleomorphic adenoma; EMC, epithelial myoepithelial carcinoma; DLBCL, diffuse large B-cell lymphoma; BCA, basal cell adenoma; MEC, mucoepidermoid carcinoma; AdCC, adenoid cystic carcinoma; Sqcc, squamous cell carcinoma; NOS, not otherwise specified.
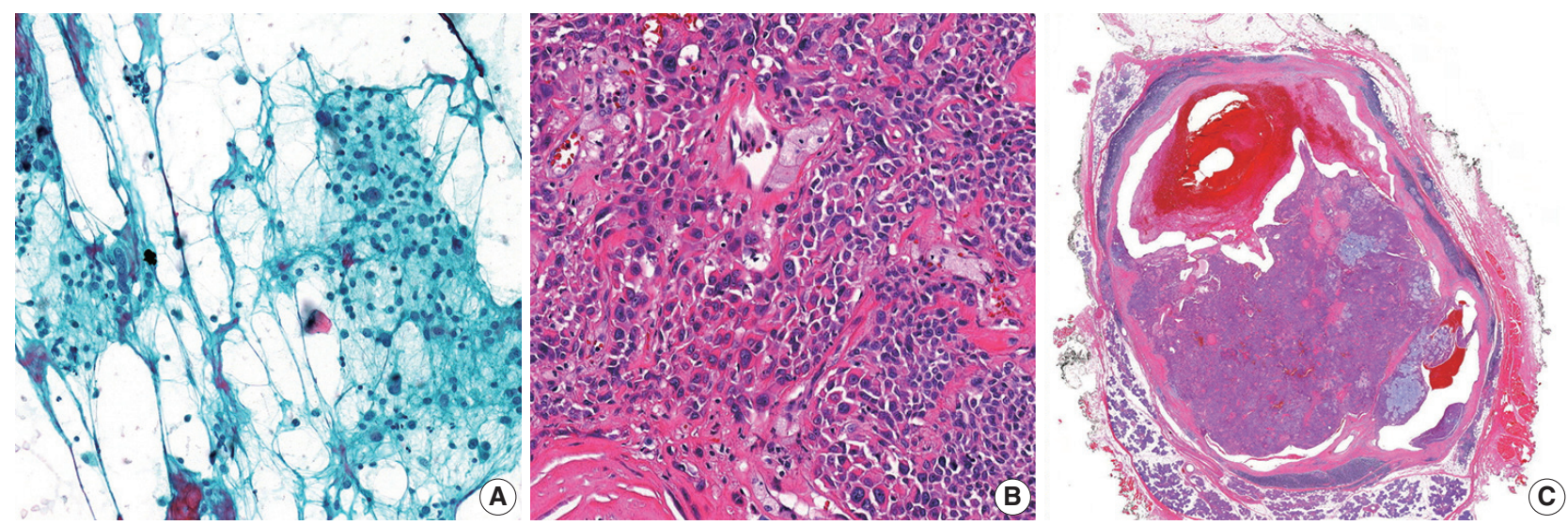

Fig. 2. Images of one of the false positive cases (atypical pleomorphic adenoma). (A) Fine-needle aspiration revealed highly atypical cells suspicious for malignant neoplasm. (B) Higher magnification of the mass showing atypical cells. (C) A lower magnification of the atypical pleomorphic adenoma without capsule invasion. 
Table 3. Stratification based on risk of malignancy (\%) in both current and previous studies

\begin{tabular}{|c|c|c|c|c|c|c|c|}
\hline & ND & NN & AUS & $\mathrm{BN}$ & SUMP & SM & M \\
\hline ROM proposed by the MSRSGC & 25.0 & 10.0 & 20.0 & $<5$ & 35.0 & 60.0 & 90.0 \\
\hline Current study & 24.0 & 0 & 40.0 & 2.5 & 46.7 & 100 & 87.5 \\
\hline Rossi et al. [16] & 17.0 & 16.0 & 53.0 & \multicolumn{2}{|c|}{$6^{a}$} & 79.0 & 100 \\
\hline Song et al. [14] & 17.8 & 14.3 & 30.6 & 2.2 & 46.6 & 78.9 & 98.8 \\
\hline Viswanathan et al. [15] & 6.7 & 7.1 & 38.9 & 5.0 & 34.2 & 92.9 & 92.3 \\
\hline Thiryayi et al. [19] & 8.5 & 1.6 & 0 & 1.9 & 26.7 & 100 & 100 \\
\hline Rohilla et al. [17] & 0 & 17.4 & 100 & 7.3 & 50.0 & - & 96.0 \\
\hline Park et al. [18] & 19.5 & 6.9 & 0 & 2.4 & 26.2 & 83.3 & 100 \\
\hline
\end{tabular}

ND, non-diagnostic; NN, non-neoplastic; AUS, atypia of undetermined significance; BN, benign neoplasm; SUMP, salivary gland neoplasm of uncertain malignant potential; SM, suspicious for malignancy; M, malignant; ROM, risk of malignancy; MSRSGC, Milan System for Reporting Salivary Gland Cytopathology. ${ }^{a}$ Cases in the BN and SUMP categories were calculated together in this study.

Table 4. Cases showing a discrepancy between preoperative FNA diagnosis according to the MSRSGC system and the final pathologic diagnosis

\begin{tabular}{llll}
\hline No. & $\begin{array}{c}\text { Type of } \\
\text { discrepancy }\end{array}$ & $\begin{array}{c}\text { MSRSGC } \\
\text { category }\end{array}$ & \multicolumn{1}{c}{$\begin{array}{c}\text { Final pathologic } \\
\text { diagnosis }\end{array}$} \\
\hline 1 & False-negative & IV-A & Mucoepidermoid carcinoma, low-grade \\
2 & False-negative & IV-A & Epithelial myoepithelial carcinoma \\
3 & False-negative & II & Warthin tumor \\
4 & False-negative & II & Sialolipoma \\
5 & False positive & VI & Atypical pleomorphic adenoma \\
6 & False positive & IV-B & Reactive lymph node \\
\hline
\end{tabular}

FNA, fine-needle aspiration; MSRSGC, Milan System for Reporting Salivary Gland Cytopathology.

samples was $95.9 \%$ (142/148 cases).

\section{DISCUSSION}

In this study, we reclassified FNA cases from a single center using the MSRSGC and correlated the results with those obtained using pathological diagnoses. Most salivary gland FNA cases that completed surgical follow-up were benign (103 cases; 69.6\%), which is similar to the incidence reported by other studies [14-17]. The diagnosis rate of ND at our institution was $23.5 \%$, with 68.0\% RON and 24.0\% ROM. Notably, the frequency of ND diagnosis was higher than the $10 \%$ frequency set by the MSRSGC.

The ROM of ND in our study was high compared to that of other studies [14-19]. Many factors are involved in an ND diagnosis, including aspiration technique, character of the lesion, method of specimen processing, and presence of artifacts from slide preparation [20]. Previous studies have suggested that rare, highly atypical cells could be placed in a category $\mathrm{V}$ if there is enough clinical suspicion, even if the number of cells to support this classification is insufficient [20].

In this study, we divided category I cases into subtypes and examined their characteristics. Interestingly, $12.5 \%$ (4/32) of all Warthin tumors were placed in category I, and all four of these cases had only cystic contents. It is presumed that Warthin tumors could be accompanied by cystic degenerative changes in the center of the lesion. When category I was divided into two subtypes (inadequate and cystic contents only), there were no significant differences between the ROM values of the two subtypes (25\% and $23.1 \%$, respectively).

The ROM values for the $\mathrm{NN}$ and $\mathrm{BN}$ categories in our study were $0 \%$ and $2.5 \%$, respectively, while the RON values were $50.0 \%$ and $100 \%$. The ROM values for the NN and BN categories were lower than the proposed ROM incidence of $10 \%$ and lower than the 5\% ROM proposed by the MSRSGC. In comparison, the ROM values for the $\mathrm{NN}$ and $\mathrm{BN}$ categories in other studies ranged from $1.6 \%-17.4 \%$ and from $1.9 \%-7.3 \%$, respectively (Table 3) [14-19]. In our study, Warthin tumors and sialolipoma cases were reported in the NN category, which is in contrast to the findings of studies by Rossi et al. [16], Viswanathan et al. [15], and Song et al. [14], in which B-cell lymphomas predominantly accounted for false-negative diagnoses. The increase in ROM in the BN category in our study was attributed to one case of low-grade mucoepidermoid carcinoma and one case of epithelial myoepithelial carcinoma, which exhibited a similar distribution to that observed in the study by Rossi et al. [16]. Moreover, in the study by Song et al., the increase in ROM in the BN category was attributed to three cases of carcinoma ex pleomorphic adenoma and one case of adenoid cystic carcinoma [14].

According to the MSRSGC guidelines, cases in the AUS category should not exceed $10 \%$ of the total cases examined. In the current study, $3.8 \%$ of salivary gland FNAs were categorized as AUS, which is in accordance with this recommendation. The ROM for the AUS category was $40 \%$, which is higher than the 20\% proposed by the MSRSGC. However, one limitation of our study was that only five AUS FNAs were included, 
precluding precise analysis of ROM for this type. In comparison, other studies have reported an ROM ranging from $0-100 \%$. Moreover, various entities were included in this category, with pleomorphic adenoma being the most common.

The RON and ROM values for the SUMP category in our study were $93.3 \%$ and $46.7 \%$, respectively, with pleomorphic adenoma and mucoepidermoid carcinoma being the most common benign and malignant diagnoses, respectively. The ROM was higher than the MSRSGC target rate and was similar to those reported in other studies (Song et al. [14] and Viswanathan et al. [15] reported ROMs of $46.6 \%$ and $34.2 \%$, respectively). For the SM category, the RON and ROM values were $100 \%$ and $100 \%$, respectively. Notably, the reported ROM for this category varies from institution to institution, ranging from 78.9\%-100\% [14-19]. This outcome is likely due to different institutional practices and disease populations, as well as differences in pathologist experience. Thus, this finding may represent a limitation of single-center studies. The RON and ROM values for the $\mathrm{M}$ category were $100 \%$ and $87.5 \%$, respectively. The ROM in our study was slightly lower compared to that in the MSRSGC and other published studies [14-19]. In previous studies, squamous cell carcinoma was the most common malignant tumor in the $\mathrm{M}$ category [14-16]. However, the $\mathrm{M}$ category in our study consisted of only one case with squamous cell carcinoma; instead, mucoepidermoid carcinoma was the most frequently diagnosed malignancy. Compared to other studies, the limited sample size (eight cases) in our study may have led to both a lower ROM and greater differences in tumor type [14-19].

In this study, there were four false-negative and two false positive cases. Contributing factors might have included sampling errors, inadequacy of technique, vagueness in interpretation, and underestimation of low-grade malignant tumors. In particular, mucoepidermoid carcinomas with cystic changes are difficult to diagnose due to a high incidence of failure to gain optimal material $[21,22]$. A limited number of mucoepidermoid carcinoma cases contains all three cell types (mucous, intermediate, and squamous cells) [23]. However, among malignant tumors, mucoepidermoid carcinoma could often be relatively straightforwardly assigned to the SM category when mucus cells are present. One of the false positive cases in the present study involved an atypical pleomorphic adenoma; the FNA specimen showed several clusters composed of markedly atypical cells in the degenerated background (Fig. 2). In the resected specimens, except for focal cytologic atypia, there were no features that indicated malignancy. Rohilla et al. [17] reported a similar false-positive case. Given this intriguing case, careful consideration of both the radiological findings and clinical assessments may help improve the predictive power of the MSRSGC.

In conclusion, we confirmed that the ROM at our institute was similar to the proposed ROM. Thus, the MSRSGC appeared to be effective in facilitating communication between pathologists and clinicians and may lead to a more comprehensive understanding of cytological diagnoses and establishment of appropriate treatment strategies.

\section{Ethics Statement}

This retrospective study was approved by the Institutional Review Board of Gangnam Severance Hospital (approval No. 3-2019-0219) with a waiver of informed consent due to its retrospective nature.

\section{ORCID}

Ji Hyun Park Yoon Jin Cha

Ja Yeong Seo

Jae Yol Lim

Soon Won Hong

\section{Author Contributions}

Conceptualization: SWH. Data curation: JHP, YJC, JYL, SWH. Formal analysis: JHP, YJC. Investigation: JHP, YJC, JYL, SWH. Methodology: JHP, JYS, YJC, JYL, SWH. Project administration: SWH. Resources: JHP, JYS, YJC, JYL, SWH. Supervision: SWH. Validation: JHP, YJC, JYL, SWH. Visualization: JHP. Writing—original draft: JHP, SWH. Writing—review \& editing: JHP, YJC, JYL, SWH.

\section{Conflicts of Interest}

SWH, a contributing editor of the Journal of Pathology and Translational Medicine, was not involved in the editorial evaluation or decision to publish this article. All remaining authors have declared no conflicts of interest.

\section{Funding Statement}

No funding to declare.

\section{Acknowledgments}

We would like to thank Editage (www.editage.co.kr) for English language editing.

\section{References}

1. Layfield LJ, Gopez E, Hirschowitz S. Cost efficiency analysis for fine-needle aspiration in the workup of parotid and submandibular gland nodules. Diagn Cytopathol 2006; 34: 734-8.

2. Mairembam P, Jay A, Beale T, et al. Salivary gland FNA cytology: role as a triage tool and an approach to pitfalls in cytomorphology. Cytopathology 2016; 27: 91-6.

3. Wang H, Fundakowski C, Khurana JS, Jhala N. Fine-needle aspiration biopsy of salivary gland lesions. Arch Pathol Lab Med 2015; 139: 1491-7.

4. Seethala RR, Griffith CC. Molecular pathology: predictive, prognostic, and diagnostic markers in salivary gland tumors. Surg Pathol Clin 2016; 9: 339-52. 
5. Pusztaszeri MP, Garcia JJ, Faquin WC. Salivary gland FNA: new markers and new opportunities for improved diagnosis. Cancer Cytopathol 2016; 124: 307-16.

6. Pusztaszeri MP, Faquin WC. Update in salivary gland cytopathology: Recent molecular advances and diagnostic applications. Semin Diagn Pathol 2015; 32: 264-74.

7. Goyal S, Sharma S, Diwaker P. Diagnostic role and limitations of FNAC in oral and jaw swellings. Diagn Cytopathol 2015; 43: 810-8.

8. Schindler S, Nayar R, Dutra J, Bedrossian CW. Diagnostic challenges in aspiration cytology of the salivary glands. Semin Diagn Pathol 2001; 18: 124-46.

9. Ashraf A, Shaikh AS, Kamal F, Sarfraz R, Bukhari MH. Diagnostic reliability of FNAC for salivary gland swellings: a comparative study. Diagn Cytopathol 2010; 38: 499-504.

10. Jain R, Gupta R, Kudesia M, Singh S. Fine needle aspiration cytology in diagnosis of salivary gland lesions: a study with histologic comparison. Cytojournal 2013; 10: 5.

11. Colella G, Cannavale R, Flamminio F, Foschini MP. Fine-needle aspiration cytology of salivary gland lesions: a systematic review. J Oral Maxillofac Surg 2010; 68: 2146-53.

12. Rossi ED, Baloch Z, Pusztaszeri M, Faquin WC. The Milan System for Reporting Salivary Gland Cytopathology (MSRSGC): an ASCIAC-sponsored system for reporting salivary gland fine-needle aspiration. J Am Soc Cytopathol 2018; 7: 111-8.

13. Rossi ED, Faquin WC, Baloch Z, et al. The Milan System for Reporting Salivary Gland Cytopathology: analysis and suggestions of initial survey. Cancer Cytopathol 2017; 125: 757-66.

14. Song SJ, Shafique K, Wong LQ, LiVolsi VA, Montone KT, Baloch Z. The utility of the Milan System as a risk stratification tool for salivary gland fine needle aspiration cytology specimens. Cytopathology 2019; 30: 91-8.
15. Viswanathan K, Sung S, Scognamiglio T, Yang GC, Siddiqui MT, Rao RA. The role of the Milan System for Reporting Salivary Gland Cytopathology: a 5-year institutional experience. Cancer Cytopathol 2018; 126: 541-51.

16. Rossi ED, Wong LQ, Bizzarro T, et al. The impact of FNAC in the management of salivary gland lesions: institutional experiences leading to a risk-based classification scheme. Cancer Cytopathol 2016; 124: 388-96.

17. Rohilla M, Singh P, Rajwanshi A, et al. Three-year cytohistological correlation of salivary gland FNA cytology at a tertiary center with the application of the Milan system for risk stratification. Cancer Cytopathol 2017; 125: 767-75.

18. Park W, Bae H, Park MH, et al. Risk of high-grade malignancy in parotid gland tumors as classified by the Milan System for Reporting Salivary Gland Cytopathology. J Oral Pathol Med 2019; 48: 222-31.

19. Thiryayi SA, Low YX, Shelton D, Narine N, Slater D, Rana DN. A retrospective 3-year study of salivary gland FNAC with categorisation using the Milan reporting system. Cytopathology 2018; 29: 343-8.

20. Faquin WC, Rossi ED, Baloch Z, et al. The Milan System for Reporting Salivary Gland Cytopathology. New York: Springer, 2018.

21. Edwards PC, Wasserman P. Evaluation of cystic salivary gland lesions by fine needle aspiration: an analysis of 21 cases. Acta Cytol 2005; 49: 489-94.

22. Frable MA, Frable WJ. Fine-needle aspiration biopsy of salivary glands. Laryngoscope 1991; 101: 245-9.

23. Joseph TP, Joseph CP, Jayalakshmy PS, Poothiode U. Diagnostic challenges in cytology of mucoepidermoid carcinoma: report of 6 cases with histopathological correlation. J Cytol 2015; 32: 21-4. 